\title{
Harvesting on Facultative Mutualist Prey Species in Presence of
}

\section{a Predator}

\author{
Saroj Kumar Chattopadhyay ${ }^{1 *}$ \\ ${ }^{1}$ Principal, Chandraketugarh Sahidullah Smriti Mahavidyalaya Berachampa, North 24-Parganas, West \\ Bengal, India \\ * Saroj Kumar Chattopadhyay, E-mail: saroj.scc@ gmail.com
}

Received: January 15, 2017

Accepted: January 24, 2017 Online Published: February 5, 2017

doi:10.22158/asir.v1n1p1

URL: http://dx.doi.org/10.22158/asir.v1n1p1

\begin{abstract}
This paper proposes a model with two preys of facultative mutualist type and one predator. Linear predation functions are considered and preys are only considered to be harvested. The stability of the model is analyzed theoretically and numerically in this paper. The optimal harvest policy is studied and the solution is derived in the interior equilibrium case using Pontryagin's maximum principle. Finally, some numerical simulations are discussed.
\end{abstract}

\section{Keywords}

mutualism, facultative, obligate, stability, optimal equilibrium

\section{Introduction}

Mutualism is an interaction in which species help one another. Janzen (1985) has argued that most of the mutualisms can be classified into one of the four classes: seed-dispersal mutualism, pollination mutualisms, digestive mutualisms, and protective mutualisms. In this paper we are interested to discuss the protective mutualisms of fish species. This type of mutualisms may be facultative mutualism or obligate mutualism. In facultative mutualism the interaction between the species is helpful but not essential but in obligate mutualism neither mutualist can survive without the other. Many fish species form protective mutualisms. A particularly well-known example involves tropical anemone fishes, or clown fishes, and their anemones. Clown fishes are immune to stinging nematocysts of giant sea anemones and will and nest amongst their tentacles. Horse mackerels appear to have a similar relationship with Portuguese man-of war jellyfish.

In recent past many works on mutualism have done (Lengeler et al., 1999; Wallin, 1923, 1927; Margulis, 1970, 1981). Those are not harvesting models. Simultaneously, some extraordinary harvesting models are studied by Clark $(1985,1990)$ and some other ecologists and scientists (Mesteron-Gibbons, 1998; Kot, 2001; Kar \& Chaudhuri, 2004; Strobele \& Wacker, 1995; Dai \& Tang, 
1998) also studied the management and behaviour dynamic harvesting models. Mark Kot (2001) discussed a two species protective mutualism model. In which there are two species with population sizes $N_{1}$ and $N_{2}$, and each species grows logistically in the absence of other. The model is

$$
\begin{aligned}
& \frac{d N_{1}}{d t}=r_{1} N_{1}\left(1-\frac{N_{1}-\alpha_{12} N_{2}}{k_{1}}\right) \\
& \frac{d N_{2}}{d t}=r_{2} N_{2}\left(1-\frac{N_{2}-\alpha_{21} N_{1}}{k_{2}}\right)
\end{aligned}
$$

where $\alpha_{12}$ and $\alpha_{21}$ are the measures of the strength of positive effect of species 2 on the species 1 and of species 1 on species 2. This model is for facultative mutualism so far $r_{1}, r_{2}, k_{1}, k_{2}$ are all positive. That is each species can, in other words, survive without its mutualist. The species may surpass their carrying capacity or may undergo unlimited growth what has been called "an orgy of mutual benefaction" (May, 1981) depending upon the values of the strength of one species on another. The equations (1) and (2) may be used for obligate mutualism if we take $r_{1}, r_{2}, k_{1}, k_{2}$ all negative. In this case neither species can survive on its own; each species is banking on the other to save it. The facultative models are generally more stable than obligate models. We state the definition of facultative or co-operative models.

Definition 1.1: The system

$$
\begin{aligned}
& \frac{d N_{1}}{d t}=f\left(N_{1}, N_{2}\right) \\
& \frac{d N_{2}}{d t}=g\left(N_{1}, N_{2}\right)
\end{aligned}
$$

defined on $\mathrm{D} \subseteq R^{2}$ is co-operative if $\frac{\partial f}{\partial N_{2}} \geq 0, \frac{\partial g}{\partial N_{1}} \geq 0$ for all $\left(N_{1}, N_{2}\right) \in D$.

In this paper, we study the problem of harvesting two facultative species in the presence of a predator species which feeds on both the facultative prey species. The predator species is not harvested. The problem is clearly stated in the section 2 . We have examined the equilibrium of the system and the conditions of their existence in section 3. The local stability of the steady state solutions is examined in section 4. We derive an optimal harvesting policy in section 5. Numerical examples are discussed in section6. The paper ends with a brief conclusion in section 7 .

\section{Formulation of the Model}

The governing equations of our model are,

$$
\begin{gathered}
\frac{d N_{1}}{d t}=r_{1} N_{1}\left(1-\frac{N_{1}-\alpha_{12} N_{2}}{K_{1}}\right)-a_{1} N_{1} N_{3}-q_{1} E_{1} N_{1} \\
\frac{d N_{2}}{d t}=r_{2} N_{2}\left(1-\frac{N_{2}-\alpha_{21} N_{1}}{K_{2}}\right)-a_{2} N_{2} N_{3}-q_{2} E_{2} N_{2}
\end{gathered}
$$




$$
\frac{d N_{3}}{d t}=\beta_{1} a_{1} N_{1} N_{3}+\beta_{2} a_{2} N_{2} N_{3}-d N_{3}
$$

where $N_{1}, N_{2}$ are population sizes of the prey species and $N_{3}$ the population size of the predator at any time t. Here, $r_{1}(>0), r_{2}(>0)$ are intrinsic growth rate of the first two species. Since we are not making a case study in respect of a specific prey-predator community, we have opted logistic growth rate. The parameters $K_{1}(>0), K_{2}(>0)$ are carrying capacities of first two species; $a_{1}, a_{2}$, both positive, are predation rates on which the third species feeds on the first two species respectively; $E_{1}, E_{2}$ are the harvesting efforts given on the first two species, the third species is not harvested; $q_{1}, q_{2}$ (both positive) are catchability co-efficients of $N_{1}, N_{2}$ respectively. The catch rate functions $q_{1} E_{1} N_{1}$ and $q_{2} E_{2} N_{2}$ are based on CPUE (catch per unit effort) hypothesis. The parameters $\beta_{1}(>0), \beta_{2}(>0)$ are known as conversion factors and $d$ is the mortality rate of predator species. Here $\alpha_{12}$ measures the strength of the positive effect of the species $N_{2}$ on the species $N_{1}$ and $\alpha_{21}$ measures the strength of the positive effect of the species $N_{1}$ on the species $N_{2}$. Considering the definition (1.1) we see that in absence of predator

$$
\frac{\partial f}{\partial N_{2}}=\frac{r_{1} \alpha_{12}}{K_{1}} N_{2} \geq 0, \text { and } \frac{\partial g}{\partial N_{1}}=\frac{r_{2} \alpha_{21}}{K_{2}} N_{1} \geq 0 .
$$

Thus our model is co-operative. We hypothetically consider that the predator does not disturb the facultative mutualism of prey species.

\section{The Equilibria of the Model and the Existence Conditions}

The biological equilibria or the steady state solutions are obtained by solving $\dot{N}_{1}=0, \dot{N}_{2}=0$, $\dot{N}_{3}=0$ where $\dot{N}_{i}(i=1,2,3)$ are time derivatives of $N_{i}(i=1,2,3)$ respectively. Solving these equations we get the points $P_{0}, P_{1}, P_{2}, P_{3}, P_{4}, P_{5}, P_{6}$ of equilibrium. The co-ordinates of these points and corresponding conditions of existence are given bellow.

The point $P_{\mathrm{O}}(\mathrm{O}, \mathrm{O}, \mathrm{O})$ is trivial which always exists, the point

$$
\begin{gathered}
P_{1}\left(0, \frac{d}{\beta_{2} a_{2}}, \frac{r_{2}}{a_{2}}\left(1-\frac{d}{K_{2} \beta_{2} a_{2}}\right)-\frac{q_{2} E_{2}}{a_{2}}\right) \text { exists if } r_{2}-q_{2} E_{2}>\frac{d r_{2}}{K_{2} \beta_{2} a_{2}}, \\
P_{2}\left(0, \frac{K_{2}}{r_{2}}\left(r_{2}-q_{2} E_{2}\right), 0\right) \text { exists if } r_{2}-q_{2} E_{2}>0, \\
P_{3}\left(\frac{K_{1}}{r_{1}}\left(r_{1}-q_{1} E_{1}\right), 0,0\right) \text { exists if } r_{1}-q_{1} E_{1}>0, \\
P_{4}\left(\frac{d}{\beta_{1} a_{1}}, 0, \frac{r_{1}}{a_{1}}\left(1-\frac{d}{K_{1} \beta_{1} a_{1}}\right)-\frac{q_{1} E_{1}}{a_{1}}\right) \text { exists if } r_{1}-q_{1} E_{1}>\frac{d r_{1}}{K_{1} \beta_{1} a_{1}}, \\
P_{5}\left(\bar{N}_{1}, \bar{N}_{2}, 0\right) \text { where } \bar{N}_{1}=\frac{1}{1-\alpha_{12} \alpha_{21}}\left[\frac{K_{1}}{r_{1}}\left(r_{1}-q_{1} E_{1}\right)+\frac{K_{2}}{r_{2}} \alpha_{12}\left(r_{2}-q_{2} E_{2}\right)\right] \\
\bar{N}_{2}=\frac{1}{1-\alpha_{12} \alpha_{21}}\left[\frac{K_{1}}{r_{1}} \alpha_{21}\left(r_{1}-q_{1} E_{1}\right)+\frac{K_{2}}{r_{2}}\left(r_{2}-q_{2} E_{2}\right)\right] \\
\text { Published by SCHOLINK INC.} \text {, and it exists if }
\end{gathered}
$$




$$
r_{1}-q_{1} E_{1}>0, r_{2}-q_{2} E_{2}>0 \text { and } \alpha_{12} \alpha_{21}<1 .
$$

The other equilibrium point is interior equilibrium and is $P_{6}\left(N_{1}^{*}, N_{2}^{*}, N_{3}^{*}\right)$

where

$$
\begin{gathered}
N_{1}^{*}=\frac{a_{2}^{2}\left(r_{1} \beta_{2}-q_{1} E_{1} \beta_{2}\right)+\frac{r_{1}}{K_{1}} \alpha_{12} d a_{2}-a_{1} a_{2}\left(r_{2} \beta_{2}-q_{2} E_{2} \beta_{2}\right)+\frac{r_{2}}{K_{2}} a_{1} d}{a_{1} a_{2}\left(\frac{r_{1}}{K_{1}} \beta_{1} \alpha_{12}+\frac{r_{2}}{K_{2}} \beta_{2} \alpha_{21}\right)+\frac{r_{1}}{K_{1}} \beta_{2} a_{2}^{2}+\frac{r_{2}}{K_{2}} \beta_{1} a_{1}^{2}} \\
N_{2}^{*}=\frac{a_{1}^{2}\left(r_{2} \beta_{1}-q_{2} E_{2} \beta_{1}\right)+\frac{r_{2}}{K_{2}} \alpha_{21} d a_{1}-a_{1} a_{2}\left(r_{1} \beta_{1}-q_{1} E_{1} \beta_{1}\right)+\frac{r_{1}}{K_{1}} a_{2} d}{a_{1} a_{2}\left(\frac{r_{1}}{K_{1}} \beta_{1} \alpha_{12}+\frac{r_{2}}{K_{2}} \beta_{2} \alpha_{21}\right)+\frac{r_{1}}{K_{1}} \beta_{2} a_{2}^{2}+\frac{r_{2}}{K_{2}} \beta_{1} a_{1}^{2}} \\
N_{3}^{*}=\frac{\left(r_{1}-q_{1} E_{1}\right)\left(\frac{r_{2}}{K_{2}} \alpha_{21} \beta_{2} a_{2}+\frac{r_{2}}{K_{2}} \beta_{1} a_{1}\right)+\left(r_{2}-q_{2} E_{2}\right)\left(\frac{r_{1}}{K_{1}} \beta_{2} a_{2}+\frac{r_{1}}{K_{1}} \alpha_{12} \beta_{1} a_{1}\right)-\frac{d r_{1} r_{2}}{K_{1} K_{2}}\left(1-\alpha_{12} \alpha_{21}\right)}{a_{1} a_{2}\left(\frac{r_{1}}{K_{1}} \beta_{1} \alpha_{12}+\frac{r_{2}}{K_{2}} \beta_{2} \alpha_{21}\right)+\frac{r_{1}}{K_{1}} \beta_{2} a_{2}^{2}+\frac{r_{2}}{K_{2}} \beta_{1} a_{1}^{2}}
\end{gathered}
$$

The interior equilibrium point exists if

$$
\left.\begin{array}{l}
r_{1}>q_{1} E_{1}, r_{2}>q_{2} E_{2}, \\
\left(r_{1}-q_{1} E_{1}\right) a_{2}^{2} \beta_{2}+\frac{r_{1}}{K_{1}} \alpha_{12} d a_{2}+\frac{r_{2}}{K_{2}} d a_{1}>a_{1} a_{2} \beta_{2}\left(r_{2}-q_{2} E_{2}\right) \\
\left(r_{2}-q_{2} E_{2}\right) a_{1}^{2} \beta_{1}+\frac{r_{2}}{K_{2}} \alpha_{21} d a_{1}+\frac{r_{1}}{K_{1}} d a_{2}>a_{1} a_{2} \beta_{1}\left(r_{1}-q_{1} E_{1}\right) \\
\left(r_{1}-q_{1} E_{1}\right)\left(\alpha_{21} \beta_{2} a_{2}+\beta_{1} a_{1}\right) \frac{r_{2}}{K_{2}}+\left(r_{2}-q_{2} E_{2}\right)\left(\beta_{2} a_{2}+\alpha_{12} \beta_{1} a_{1}\right) \frac{r_{1}}{K_{1}}>\frac{d r_{1} r_{2}}{K_{1} K_{2}}\left(1-\alpha_{12} \alpha_{21}\right)
\end{array}\right\}
$$

\section{Local Stability Analysis}

To analyze the local stability at the different equilibria we consider the following community matrix and use variational principle.

$$
J=\left[\begin{array}{ccc}
v_{11} & \frac{r_{1} \alpha_{12} N_{1}}{K_{1}} & -a_{1} N_{1} \\
\frac{r_{2} \alpha_{21} N_{2}}{K_{2}} & v_{22} & -a_{2} N_{2} \\
\beta_{1} a_{1} N_{3} & \beta_{2} a_{2} N_{3} & \beta_{1} a_{1} N_{1}+\beta_{2} a_{2} N_{2}-d
\end{array}\right]
$$

where

$$
\begin{aligned}
& v_{11}=r_{1}\left[1-\frac{N_{1}-\alpha_{12} N_{2}}{K_{1}}\right]-a_{1} N_{3}-q_{1} E_{1}-\frac{r_{1} N_{1}}{K_{1}} \\
& v_{22}=r_{2}\left[1-\frac{N_{2}-\alpha_{21} N_{1}}{K_{2}}\right]-a_{2} N_{3}-q_{2} E_{2}-\frac{r_{2} N_{2}}{K_{2}}
\end{aligned}
$$

At $P_{0}(0,0,0)$ the community matrix reduces to

$$
J_{0}=\left[\begin{array}{ccc}
r_{1}-q_{1} E_{1} & 0 & 0 \\
0 & r_{2}-q_{2} E_{2} & 0 \\
0 & 0 & -d
\end{array}\right]
$$


whose eigenvalues are $\lambda_{0}^{1}=r_{1}-q_{1} E_{1}, \lambda_{0}^{2}=r_{2}-q_{2} E_{2}, \lambda_{0}^{3}=-d$. Thus origin is a stable node if $E_{1}>\frac{r_{1}}{q_{1}}, E_{2}>\frac{r_{2}}{q_{2}}$ that is if the harvesting efforts are more than the corresponding biotechnical productivity of the two facultative prey species. We also see that if the system be stabilized at origin then no other equilibrium of the system will be found.

At $P_{1}\left(0, \frac{d}{\beta_{2} a_{2}}, \frac{r_{2}}{a_{2}}\left(1-\frac{d}{K_{2} \beta_{2} a_{2}}\right)-\frac{q_{2} E_{2}}{a_{2}}\right)$ the Jacobian matrix reduces to

$$
J_{1}=\left[\begin{array}{ccc}
r_{1}\left(1+\frac{\alpha_{12} d}{K_{1} \beta_{2} a_{2}}\right)-q_{1} E_{1}-a_{1} \bar{z}_{1} & 0 & 0 \\
\frac{r_{2} \alpha_{21} d}{K_{2} \beta_{2} a_{2}} & \frac{-r_{2} d}{K_{2} \beta_{2} a_{2}} & \frac{-d}{\beta_{2}} \\
\beta_{1} a_{1} \bar{z}_{1} & \beta_{2} a_{2} \bar{z}_{1} & 0
\end{array}\right]
$$

Where $\quad \bar{z}_{1}=\frac{r_{2}}{a_{2}}\left(1-\frac{d}{K_{2} \beta_{2} a_{2}}\right)-\frac{q_{2} E_{2}}{a_{2}}$. One of the eigenvalues of $J_{1}$ is $\lambda_{1}^{1}=r_{1}\left(1+\frac{\alpha_{12} d}{K_{1} \beta_{2} a_{2}}\right)-q_{1} E_{1}-a_{1} \bar{z}_{1}$ which may be positive or negative depending upon the values of the parameters. In the inspection of other two eigenvalues we see that they are the roots of the quadratic

$$
\lambda^{2}+\frac{r_{2} d}{K_{2} \beta_{2} a_{2}} \lambda-d a_{2} \bar{z}_{1}=0
$$

The sum of whose roots $=-\frac{r_{2} d}{K_{2} \beta_{2} a_{2}}<0$, the product of the roots= $-d a_{2} \bar{z}_{1}<0$ with the assumption that $P_{1}$ exists. Thus other two eigenvalues are one positive and one negative. Hence $P_{1}$ is an unstable equilibrium, whatever the sign of $\lambda_{1}^{1}=r_{1}\left(1+\frac{\alpha_{12} d}{K_{1} \beta_{2} a_{2}}\right)-q_{1} E_{1}-a_{1} \bar{z}_{1}$ may be. At $P_{2}\left(\mathrm{O}, \frac{K_{2}}{r_{2}}\left(r_{2}-q_{2} E_{2}\right), \mathrm{O}\right)$ the community matrix becomes

$$
J_{2}=\left[\begin{array}{ccc}
r_{1}\left(1+\frac{K_{2} \alpha_{12}}{K_{1} r_{2}}\left(r_{2}-q_{2} E_{2}\right)\right)-q_{1} E_{1} & 0 & 0 \\
\alpha_{21}\left(r_{2}-q_{2} E_{2}\right) & -\left(r_{2}-q_{2} E_{2}\right) & -\frac{a_{2} K_{2}}{r_{2}}\left(r_{2}-q_{2} E_{2}\right) \\
0 & 0 & \frac{\beta_{2} a_{2} K_{2}}{r_{2}}\left(r_{2}-q_{2} E_{2}\right)-d
\end{array}\right] .
$$

Whose eigenvalues are,

$$
\begin{gathered}
\lambda_{2}^{1}=\left(r_{1}-q_{1} E_{1}\right)+\frac{r_{1} K_{2}}{r_{2} K_{1}} \alpha_{12}\left(r_{2}-q_{2} E_{2}\right), \\
\lambda_{2}^{2}=-\left(r_{2}-q_{2} E_{2}\right), \\
\lambda_{2}^{1}=\frac{\beta_{2} K_{2}}{r_{2}} a_{2}\left(r_{2}-q_{2} E_{2}\right)-d .
\end{gathered}
$$


Now, when $P_{2}$ exists, $\left(r_{2}-q_{2} E_{2}\right)>0$. Thus the above eigenvalues are negative i.e., the equilibrium at $P_{2}$ is asymptotically stable if

$$
0<\left(r_{2}-q_{2} E_{2}\right)<\operatorname{Min}\left\{\frac{r_{2} d}{\beta_{2} K_{2} a_{2}}, \frac{r_{2} K_{1}}{r_{1} K_{2} \alpha_{12}}\left(q_{1} E_{1}-r_{1}\right)\right\} \text { and }\left(r_{1}-q_{1} E_{1}\right)<0
$$

At $P_{3}\left(\frac{K_{1}}{r_{1}}\left(r_{1}-q_{1} E_{1}\right), 0,0\right)$ the Jacobian matrix (15) reduces to

$$
J_{3}=\left[\begin{array}{ccc}
-\left(r_{1}-q_{1} E_{1}\right) & \alpha_{12}\left(r_{1}-q_{1} E_{1}\right) & -\frac{a_{1} K_{1}}{r_{1}}\left(r_{1}-q_{1} E_{1}\right) \\
0 & r_{2}\left(1+\frac{\alpha_{21} K_{1}}{K_{2} r_{1}}\left(r_{1}-q_{1} E_{1}\right)\right)-q_{2} E_{2} & 0 \\
0 & 0 & \frac{\beta_{1} a_{1} K_{1}}{r_{1}}\left(r_{1}-q_{1} E_{1}\right)-d
\end{array}\right]
$$

whose eigenvalues are,

$$
\begin{gathered}
\lambda_{3}^{1}=-\left(r_{1}-q_{1} E_{1}\right)<0, \\
\lambda_{3}^{2}=\left(r_{2}-q_{2} E_{2}\right)+\frac{r_{2} K_{1}}{r_{1} K_{2}} \alpha_{21}\left(r_{1}-q_{1} E_{1}\right), \\
\lambda_{3}^{3}=\frac{\beta_{1} K_{1}}{r_{1}} a_{1}\left(r_{1}-q_{1} E_{1}\right)-d .
\end{gathered}
$$

Thus the eigenvalues are negative real numbers that is the equilibrium at $P_{3}$ is asymptotically stable if

$$
0<\left(r_{1}-q_{1} E_{1}\right)<\operatorname{Min}\left\{\frac{r_{1} d}{\beta_{1} K_{1} a_{1}}, \frac{r_{1} K_{2}}{r_{2} K_{1} \alpha_{21}}\left(q_{2} E_{2}-r_{2}\right)\right\} \text { and }\left(r_{2}-q_{2} E_{2}\right)<0 .
$$

At $P_{4}\left(\frac{d}{\beta_{1} a_{1}}, 0, \frac{r_{1}}{a_{1}}\left(1-\frac{d}{K_{1} \beta_{1} a_{1}}\right)-\frac{q_{1} E_{1}}{a_{1}}\right) \equiv P_{4}\left(\bar{x}_{4}, 0, \bar{z}_{4}\right)$, say, the Jacobian matrix (15) becomes

$$
J_{4}=\left[\begin{array}{ccc}
-\frac{r_{1} d}{K_{1} \beta_{1} a_{1}} & \frac{r_{1} d \alpha_{12}}{K_{1} \beta_{1} a_{1}} & -\frac{d}{\beta_{1}} \\
0 & r_{2}\left(1+\frac{\alpha_{21} d}{K_{2} \beta_{1} a_{1}}\right)-q_{2} E_{2}-a_{2}\left(\frac{r_{1}-q_{1} E_{1}}{a_{1}}-\frac{r_{1} d}{K_{1} \beta_{1} a_{1}^{2}}\right) & 0 \\
\beta_{1} a_{1} \bar{z}_{4} & \beta_{2} a_{2} \bar{z}_{4} & 0
\end{array}\right] .
$$

One of whose eigenvalues is $\lambda_{4}^{1}=r_{2}\left(1+\frac{\alpha_{21} d}{K_{2} \beta_{1} a_{1}}\right)-q_{2} E_{2}-a_{2}\left(\frac{r_{1}-q_{1} E_{1}}{a_{1}}-\frac{r_{1} d}{K_{1} \beta_{1} a_{1}^{2}}\right)$ and other two eigenvalues are the roots of $\lambda^{2}+\frac{r_{1} d}{K_{1} \beta_{1} a_{1}} \lambda+a_{1} \bar{z}_{4} d=0$. The sum of whose roots $=-\frac{r_{1} d}{K_{1} \beta_{1} a_{1}}<0$, and product of the roots $=a_{1} \bar{z}_{4} d>0$ under the condition of existence of $P_{4}$. Thus by Routh-Hurwitz rule the equilibrium at $P_{4}$ is asymptotically stable if $\lambda_{4}^{1}<0$ when the equilibrium at $P_{4}$ exists. Thus $P_{4}$ is a stable equilibrium if,

$$
\left(r_{2}-q_{2} E_{2}\right)+\frac{r_{2} \alpha_{21} d}{K_{2} \beta_{1} a_{1}}>\frac{a_{2}}{a_{1}}\left(r_{1}-q_{1} E_{1}-\frac{r_{1} d}{K_{1} \beta_{1} a_{1}}\right) \quad \text { where } \quad\left(r_{1}-q_{1} E_{1}\right)>\frac{r_{1} d}{K_{1} \beta_{1} a_{1}}
$$

At the equilibrium point $P_{5}\left(\bar{N}_{1}, \bar{N}_{2}, 0\right)$ where 


$$
\begin{aligned}
& \bar{N}_{1}=\frac{1}{1-\alpha_{12} \alpha_{21}}\left[\frac{K_{1}}{r_{1}}\left(r_{1}-q_{1} E_{1}\right)+\frac{K_{2}}{r_{2}} \alpha_{12}\left(r_{2}-q_{2} E_{2}\right)\right], \\
& \bar{N}_{2}=\frac{1}{1-\alpha_{12} \alpha_{21}}\left[\frac{K_{1}}{r_{1}} \alpha_{21}\left(r_{1}-q_{1} E_{1}\right)+\frac{K_{2}}{r_{2}}\left(r_{2}-q_{2} E_{2}\right)\right]
\end{aligned}
$$

with $r_{1}-q_{1} E_{1}>0, \quad r_{2}-q_{2} E_{2}>0$ and $\alpha_{12} \alpha_{21}<1$, the Jacobian matrix (15) reduces to

$$
J_{5}=\left[\begin{array}{ccc}
-\frac{r_{1}}{K_{1}} \bar{N}_{1} & \frac{r_{1} \alpha_{12} \bar{N}_{1}}{K_{1}} & -a_{1} \bar{N}_{1} \\
\frac{r_{2} \alpha_{21} \bar{N}_{2}}{K_{2}} & -\frac{r_{2} \bar{N}_{2}}{K_{2}} & -a_{2} \bar{N}_{2} \\
0 & 0 & \beta_{1} a_{1} \bar{N}_{1}+\beta_{2} a_{2} \bar{N}_{2}-d
\end{array}\right]
$$

One of the eigenvalues of $J_{5}$ is $\lambda_{5}^{1}=\beta_{1} a_{1} \bar{N}_{1}+\beta_{2} a_{2} \bar{N}_{2}-d$, and other two are the roots of the quadratic

$$
\lambda^{2}+\left(\frac{r_{1} \bar{N}_{1}}{K_{1}}+\frac{r_{2} \bar{N}_{2}}{K_{2}}\right) \lambda+\left(1-\alpha_{12} \alpha_{21}\right) \frac{r_{1} r_{2}}{K_{1} K_{2}} \bar{N}_{1} \bar{N}_{2}=0
$$

The sum of whose roots $=-\left(\frac{r_{1} \bar{N}_{1}}{K_{1}}+\frac{r_{2} \bar{N}_{2}}{K_{2}}\right)<0$,

and the product of the roots $={ }^{\left(1-\alpha_{12} \alpha_{21}\right) \frac{r_{1} r_{2}}{K_{1} K_{2}} \bar{N}_{1} \bar{N}_{2}>0}$ under the conditions of existence of $P_{5}$. Thus by Routh-Hurwitz rule the equilibrium point $P_{5}$ is asymptotically stable if $\beta_{1} a_{1} \bar{N}_{1}+\beta_{2} a_{2} \bar{N}_{2}-d<0$, that is if

$$
\left[\left(r_{1}-q_{1} E_{1}\right)\left(\frac{\beta_{1} a_{1} K_{1}}{r_{1}}+\frac{\beta_{2} a_{2} K_{1}}{r_{1}} \alpha_{21}\right)+\left(r_{2}-q_{2} E_{2}\right)\left(\frac{\beta_{1} a_{1} K_{2}}{r_{2}} \alpha_{12}+\frac{\beta_{2} a_{2} K_{2}}{r_{2}}\right)\right]<d\left(1-\alpha_{12} \alpha_{21}\right)
$$

together with the condition of existence of $P_{5}$.

The community matrix at $P_{6}\left(N_{1}^{*}, N_{2}^{*}, N_{3}^{*}\right)$ is

$$
J_{6}=\left[\begin{array}{ccc}
-\frac{r_{1}}{K_{1}} N_{1}^{*} & \frac{r_{1} \alpha_{12} N_{1}^{*}}{K_{1}} & -a_{1} N_{1}^{*} \\
\frac{r_{2} \alpha_{21} N_{2}^{*}}{K_{2}} & -\frac{r_{2} N_{2}^{*}}{K_{2}} & -a_{2} N_{2}^{*} \\
\beta_{1} a_{1} N_{3}^{*} & \beta_{2} a_{2} N_{3}^{*} & 0
\end{array}\right] .
$$

The eigenvalues $\lambda_{6}^{i}(i=1,2,3)$ of $J_{6}$ are the roots of the following characteristic cubic

$$
\lambda^{3}-\text { Trace }\left|J_{6}\right| \lambda^{2}+\text { Trace } \mid \text { adj. } J_{6}|\lambda-| J_{6} \mid=0
$$

By Routh-Hurwitz rule, this cubic has roots with negative real parts if Trace $\left|J_{6}\right|<0,\left|J_{6}\right|<0$, and Trace $\left|J_{6}\right|$.Trace $\mid$ adj. $J_{6}|<| J_{6} \mid$.

Now, $\xi=\left|J_{6}\right|=-\left(N_{1}^{*} N_{2}^{*} N_{3}^{*}\right)\left[\frac{r_{1}}{K_{1}}\left(\beta_{2} a_{2}^{2}+\alpha_{12} a_{1} a_{2} \beta_{1}\right)+\frac{r_{2}}{K_{2}}\left(\alpha_{12} a_{1} a_{2} \beta_{2}+a_{1}^{2} \beta_{1}\right)\right]<0$, $\eta=\operatorname{Trace}\left|J_{6}\right|=-\left(\frac{r_{1} N_{1}^{*}}{K_{1}}+\frac{r_{2} N_{2}^{*}}{K_{2}}\right)<0$ and $\zeta=\operatorname{Trace}\left|\operatorname{adj.J_{6}}\right|=N_{2}^{*} N_{3}^{*} \beta_{2} a_{2}^{2}+N_{1}^{*} N_{3}^{*} \beta_{1} a_{1}^{2}+\frac{r_{1} r_{2}}{K_{1} K_{2}}\left(1-\alpha_{12} \alpha_{21}\right) N_{1}^{*} N_{2}^{*}$. Therefore the 
interior equilibrium at $P_{6}\left(N_{1}^{*}, N_{2}^{*}, N_{3}^{*}\right)$ is asymptotically stable if $\eta \zeta<\xi$, that is if

$$
\begin{aligned}
\frac{r_{1}}{K_{1}} \beta_{1} a_{1}^{2} N_{1}^{* 2} N_{3}^{* *}+ & \frac{r_{2}}{K_{2}} \beta_{2} a_{2}^{2} N_{2}^{* 2} N_{3}^{*}+\left(\frac{r_{1}}{K_{1}} N_{1}^{* *}+\frac{r_{2}}{K_{2}} N_{2}^{* *}\right) \frac{r_{1} r_{2}}{K_{1} K_{2}}\left(1-\alpha_{12} \alpha_{21}\right) N_{1}^{* *} N_{2}^{*} \\
& >N_{1}^{*} N_{2}^{*} N_{3}^{*} a_{1} a_{2}\left(\frac{r_{1} \beta_{1} \alpha_{12}}{K_{1}}+\frac{r_{2} \beta_{2} \alpha_{21}}{K_{2}}\right)
\end{aligned}
$$

\section{Optimal Harvesting Policy}

Once the process of harvesting the resources is started, the problem of management of the fisheries can be viewed in terms of rent maximization. Now we shall use Pontryagin's maximum principle to solve our optimization problem and obtain optimal harvest policies $E_{1}(t), E_{2}(t)$ such that our objective functional is maximized. Importance of discount rate cannot be underestimated in addressing environmental and resources issues. In fact the optimal stock size for a given fishery will vary depending on discount rate. Now we assume that

$p_{1}=$ Constant price per unit of biomass of $N_{1}-$ species,

$p_{2}=$ Constant price per unit of biomass of $N_{2}-$ species,

$c_{1}, c_{2}$ are constant costs of fishing for the two facultative prey species per unit effort.

Our objective is to study the optimal harvest policy, i.e., $E_{i}(t) \in\left[0, E_{i}^{\max }\right], i=1,2$ and $t \in[0, \propto[$ to maximize the profits of harvesting agencies and to keep the populations at an optimum level. The objective functional representing the present value $J$ of a continuous time stream of revenue is given by

$$
J\left(E_{1}, E_{2}\right)=\int_{0}^{\infty} e^{-\delta t}\left[p_{1} q_{1} E_{1} N_{1}+p_{2} q_{2} E_{2} N_{2}-c_{1} E_{1}-c_{2} E_{2}\right) d t
$$

where $\delta$ is instantaneous annual rate of discount. In this section we will find an optimal harvest policy solving the following optimization problem,

Maximize $J\left(E_{1}, E_{2}\right)$

Subject to (3)-(5)

and $\mathrm{O} \leq E_{i}(t) \leq E_{i}^{\max } ; i=1,2$

The Hamiltonian for this control problem is taken as

$$
\begin{aligned}
& \mathrm{H}=e^{-\delta t}\left[p_{1} q_{1} E_{1} N_{1}+p_{2} q_{2} E_{2} N_{2}-c_{1} E_{1}-c_{2} E_{2}\right] \\
& \left.{ }_{+} \lambda_{1} r_{1} N_{1}\left(1-\frac{N_{1}-\alpha_{12} N_{2}}{K_{1}}\right)-a_{1} N_{1} N_{3}-q_{1} E_{1} N_{1}\right] \\
& +\lambda_{2}\left[r_{2} N_{2}\left(1-\frac{N_{2}-\alpha_{21} N_{1}}{K_{2}}\right)-a_{2} N_{2} N_{3}-q_{2} E_{2} N_{2}\right] \\
& { }_{+} \lambda_{3}\left[\beta_{1} a_{1} N_{1} N_{3}+\beta_{2} a_{2} N_{2} N_{3}-d N_{3}\right]
\end{aligned}
$$

where $\lambda_{i}=\lambda_{i}(t), i=1,2,3$ are adjoint variables.

We have,

$$
\frac{\partial H}{\partial E_{1}}=e^{-\delta t}\left[p_{1} q_{1} N_{1}-c_{1}\right]-\lambda_{1} q_{1} N_{1}=\sigma_{1}(t)
$$


and

$$
\frac{\partial H}{\partial E_{2}}=e^{-\delta t}\left[p_{2} q_{2} N_{2}-c_{2}\right]-\lambda_{2} q_{2} N_{2}=\sigma_{2}(t)
$$

The optimal control $E_{i}(t), i=1,2$ must clearly satisfy the condition

$$
E_{i}(t)=\left\{E_{i}^{\max } \quad \text { when } \sigma_{i}(t)>0 \text {, and } 0 \text { when } \sigma_{i}(t)<0\right.
$$

Since $\sigma_{i}(t)$ causes $E_{i}(t)$ to switch between the levels 0 and $E^{\max }, \sigma_{i}(t){ }_{(i=1,2)}$ are called switching functions. Depending on the switching functions $\sigma_{i}(t)$, the optimal control $E_{i}(t)$ is a bang-bang control switching from one extreme level to other one. Once $\sigma_{i}(t)(i=1,2)$ vanishes ,the Hamiltonian functions $\mathrm{H}$ becomes independent of the control variable $\boldsymbol{E}_{i}(t)(i=1,2)$ and its optimal value cannot be determined by the above procedure. It is then called singular control $E_{i}^{*}(t), O<E_{i}^{*}(t)<E_{i}^{\max }$. Hence our optimal harvest policy becomes

$$
E_{i}(t)=\left\{\begin{array}{l}
E_{i}^{\max } \text { for } \sigma_{i}(t)>0 \\
0 \quad \text { for } \sigma_{i}(t)>0 \\
E_{i}^{*}(t) \text { for } \sigma_{i}=0
\end{array}\right.
$$

for $i=1,2$. For singular control $\sigma_{i}(t)=0 ; i=1,2$. Then from (26) and (27) we find that the values of $\lambda_{1}$ and $\lambda_{2}$ are

$$
\begin{aligned}
& \lambda_{1}=e^{-\delta t}\left(p_{1}-\frac{c_{1}}{q_{1} N_{1}}\right) \\
& \lambda_{2}=e^{-\delta t}\left(p_{2}-\frac{c_{2}}{q_{2} N_{2}}\right)
\end{aligned}
$$

Now the adjoint equations are

$$
\frac{d \lambda_{1}}{d t}=-\frac{\partial H}{\partial N_{1}}, \quad \frac{d \lambda_{2}}{d t}=-\frac{\partial H}{\partial N_{2}}, \quad \frac{d \lambda_{3}}{d t}=-\frac{\partial H}{\partial N_{3}}
$$

Then using (25) and the third equation of (31) we find

$$
\frac{d \lambda_{3}}{d t}=-\left[-\lambda_{1} a_{1} N_{1}-\lambda_{2} a_{2} N_{2}+\lambda_{3}\left(\beta_{1} a_{1} N_{1}+\beta_{2} a_{2} N_{2}-d\right)\right]
$$

Using (29), (30) we write,

$$
\begin{gathered}
\frac{d \lambda_{3}}{d t}=e^{-\delta t}\left(p_{1}-\frac{c_{1}}{q_{1} N_{1}}\right) a_{1} N_{1}+e^{-\delta t}\left(p_{2}-\frac{c_{2}}{q_{2} N_{2}}\right) a_{2} N_{2} \\
\lambda_{3}=\frac{e^{-\delta t}}{\delta}\left[\left(p_{1}-\frac{c_{1}}{q_{1} N_{1}}\right) a_{1} N_{1}+\left(p_{2}-\frac{c_{2}}{q_{2} N_{2}}\right) a_{2} N_{2}\right]
\end{gathered}
$$

We here consider that the constant of integration vanishes so that the shadow prices $\lambda_{i} e^{\delta t}(i=1,2,3)$ of three species are bounded. Using (29) and (30) in other two equations of (31) we find that,

$$
\delta e^{-\delta t}\left(p_{1}-\frac{c_{1}}{q_{1} N_{1}}\right)=\left[e^{-\delta \delta} p_{1} q_{1} E_{1}-\frac{\lambda_{1}}{K_{1}} N_{1} r_{1}+\frac{\lambda_{2}}{K_{2}} r_{2} N_{2} \alpha_{21}+\lambda_{3} \beta_{1} a_{1} N_{3}\right]
$$

and, 


$$
\delta e^{-\delta t}\left(p_{2}-\frac{c_{2}}{q_{2} N_{2}}\right)=\left[e^{-\delta t} p_{2} q_{2} E_{2}-\frac{\lambda_{2}}{K_{2}} N_{2} r_{2}+\frac{\lambda_{1}}{K_{1}} r_{1} N_{1} \alpha_{12}+\lambda_{3} \beta_{2} a_{2} N_{3}\right]
$$

Now using the values of the adjoint variables $\lambda_{1}, \lambda_{2}, \lambda_{3}$ in the above equations we get,

$\delta\left(p_{1}-\frac{c_{1}}{q_{1} N_{1}}\right)=\left[p_{1} q_{1} E_{1}-\frac{r_{1}}{K_{1}}\left(p_{1}-\frac{c_{1}}{q_{1} N_{1}}\right) N_{1}+\frac{r_{2}}{K_{2}}\left(p_{2}-\frac{c_{2}}{q_{2} N_{2}}\right) \alpha_{21} N_{2}-\frac{\beta_{1} a_{1} N_{3}}{\delta}\left\{a_{1}\left(p_{1}-\frac{c_{1}}{q_{1} N_{1}}\right) N_{1}+a_{2}\left(p_{2}-\frac{c_{2}}{q_{2} N_{2}}\right) N_{2}\right\}\right]$

and, $\delta\left(p_{2}-\frac{c_{2}}{q_{2} N_{2}}\right)=\left[p_{2} q_{2} E_{2}+\frac{r_{1}}{K_{1}}\left(p_{1}-\frac{c_{1}}{q_{1} N_{1}}\right) N_{1} \alpha_{12}-\frac{r_{2}}{K_{2}}\left(p_{2}-\frac{c_{2}}{q_{2} N_{2}}\right) N_{2}-\frac{\beta_{2} a_{2} N_{3}}{\delta}\left\{a_{1}\left(p_{1}-\frac{c_{1}}{q_{1} N_{1}}\right) N_{1}+a_{2}\left(p_{2}-\frac{c_{2}}{q_{2} N_{2}}\right) N_{2}\right\}\right]$

Therefore,

$$
p_{1} q_{1} E_{1}=\left(p_{1}-\frac{c_{1}}{q_{1} N_{1}}\right)\left(\delta+\frac{r_{1} N_{1}}{K_{1}}+\frac{\beta_{1} a_{1}^{2} N_{1} N_{3}}{\delta}\right)-\left(p_{2}-\frac{c_{2}}{q_{2} N_{2}}\right)\left(\frac{\beta_{1} a_{1} a_{2} N_{2} N_{3}}{\delta}-\frac{r_{2} \alpha_{21} N_{2}}{K_{2}}\right)
$$

and

$$
p_{2} q_{2} E_{2}=\left(p_{1}-\frac{c_{1}}{q_{1} N_{1}}\right)\left(\frac{\beta_{2} a_{1} a_{2} N_{1} N_{3}}{\delta}-\frac{r_{1} \alpha_{12} N_{1}}{K_{1}}\right)-\left(p_{2}-\frac{c_{2}}{q_{2} N_{2}}\right)\left(\delta+\frac{r_{2} N_{2}}{K_{2}}+\frac{\beta_{2} a_{2}^{2} N_{1} N_{3}}{\delta}\right)
$$

From these we get the following expressions of harvesting efforts,

$$
\begin{gathered}
E_{1}=\frac{1}{p_{1} q_{1}}\left[\left(p_{1}-\frac{c_{1}}{q_{1} N_{1}}\right)\left(\delta+\frac{r_{1} N_{1}}{K_{1}}+\frac{\beta_{1} a_{1}^{2} N_{1} N_{3}}{\delta}\right)-\left(p_{2}-\frac{c_{2}}{q_{2} N_{2}}\right)\left(\frac{\beta_{1} a_{1} a_{2} N_{2} N_{3}}{\delta}-\frac{r_{2} \alpha_{21} N_{2}}{K_{2}}\right)\right] \\
E_{2}=\frac{1}{p_{2} q_{2}}\left[\left(p_{1}-\frac{c_{1}}{q_{1} N_{1}}\right)\left(\frac{\beta_{2} a_{1} a_{2} N_{1} N_{3}}{\delta}-\frac{r_{1} \alpha_{12} N_{1}}{K_{1}}\right)-\left(p_{2}-\frac{c_{2}}{q_{2} N_{2}}\right)\left(\delta+\frac{r_{2} N_{2}}{K_{2}}+\frac{\beta_{2} a_{2}^{2} N_{1} N_{3}}{\delta}\right)\right]
\end{gathered}
$$

Hence solving the steady state equations together with (32) and (33) we get an optimal equilibrium solution $\left(N_{1 \delta}, N_{2 \delta}, N_{3 \delta}\right)$ and optimal harvesting efforts $E_{1 \delta}$ and $E_{2 \delta}$.

\section{Numerical Simulation}

Example 1. For numerical analysis we first consider the following set of values of parameters,

$$
\begin{aligned}
& r_{1}=2.09, K_{1}=100, \alpha_{12}=0.1, a_{1}=0.01, q_{1}=0.04, E_{1}=10, r_{2}=2.5, K_{2}=100, \\
& \alpha_{21^{\circ}}=0.2, a_{2}=0.02, q_{2}=0.05, E_{2}=12, \beta_{1}=0.3, \beta_{2}=0.4, d=0.2
\end{aligned}
$$

Then the equilibrium points are $\boldsymbol{P}_{\mathrm{O}}(0,0,0), \quad \boldsymbol{P}_{1}(0,25,63.75), \quad \boldsymbol{P}_{2}(0,76,0), \quad \boldsymbol{P}_{3}(80.86124,0,0)$, $P_{4}(66.6666,0,29.6666), \quad P_{5}(90.2666,94.0533,0), \quad P_{6}(38.2647,10.6507,91.2527)$. 


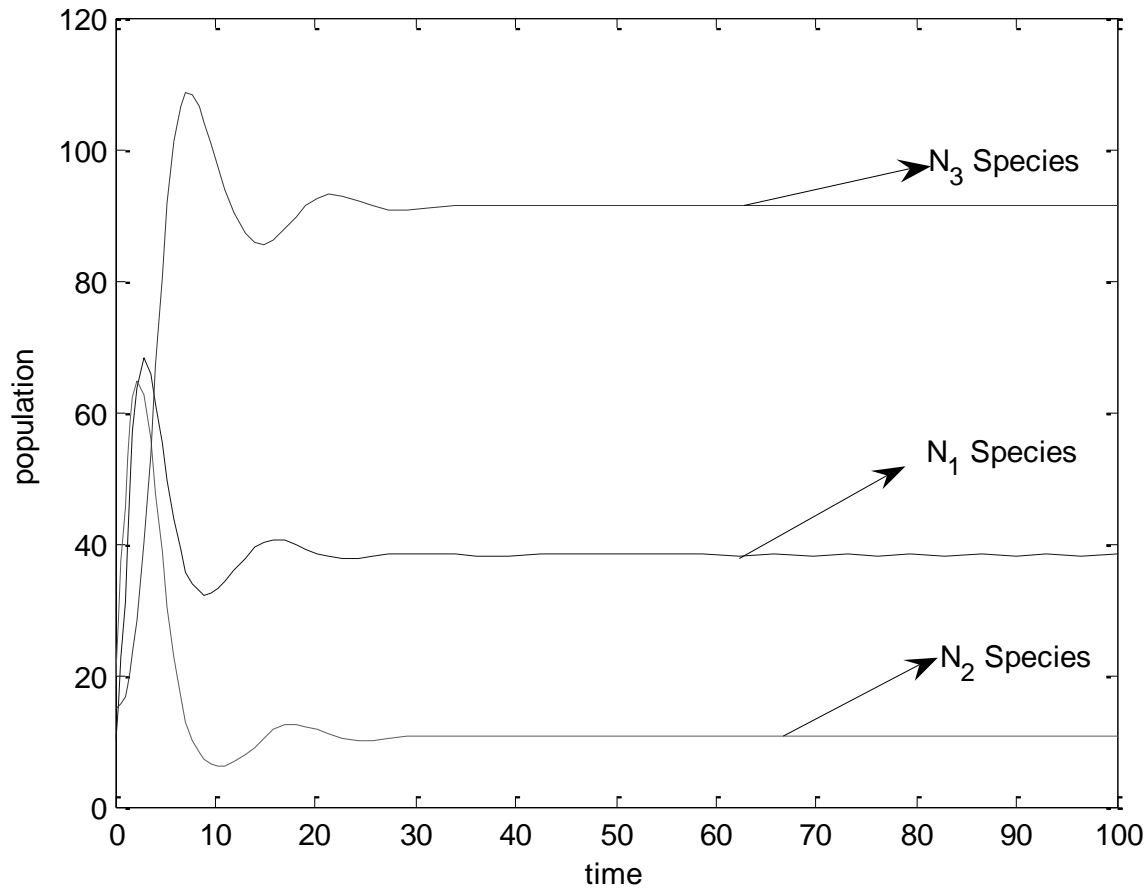

Figure 1. Solution Curves of the Species

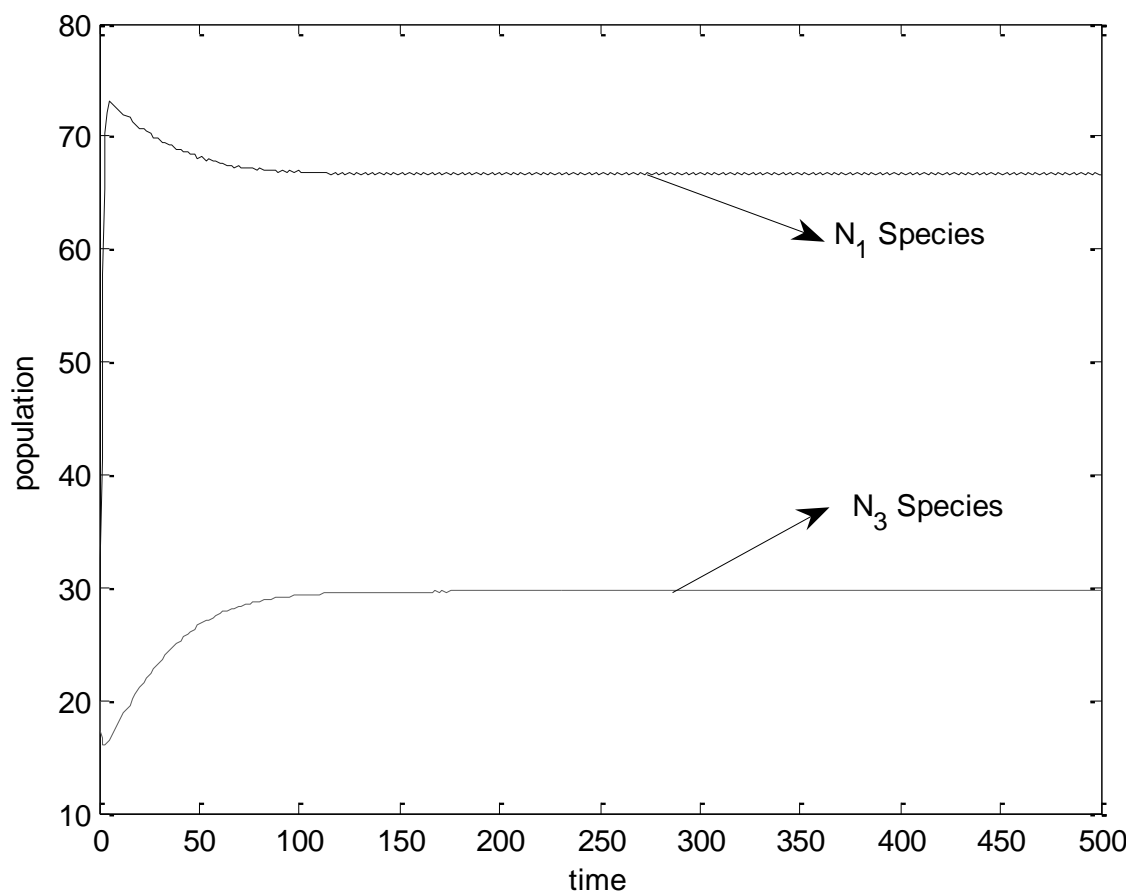

Figure 2. Solution Curves When the Prey Species $\mathbf{N}_{2}$ is Absent 


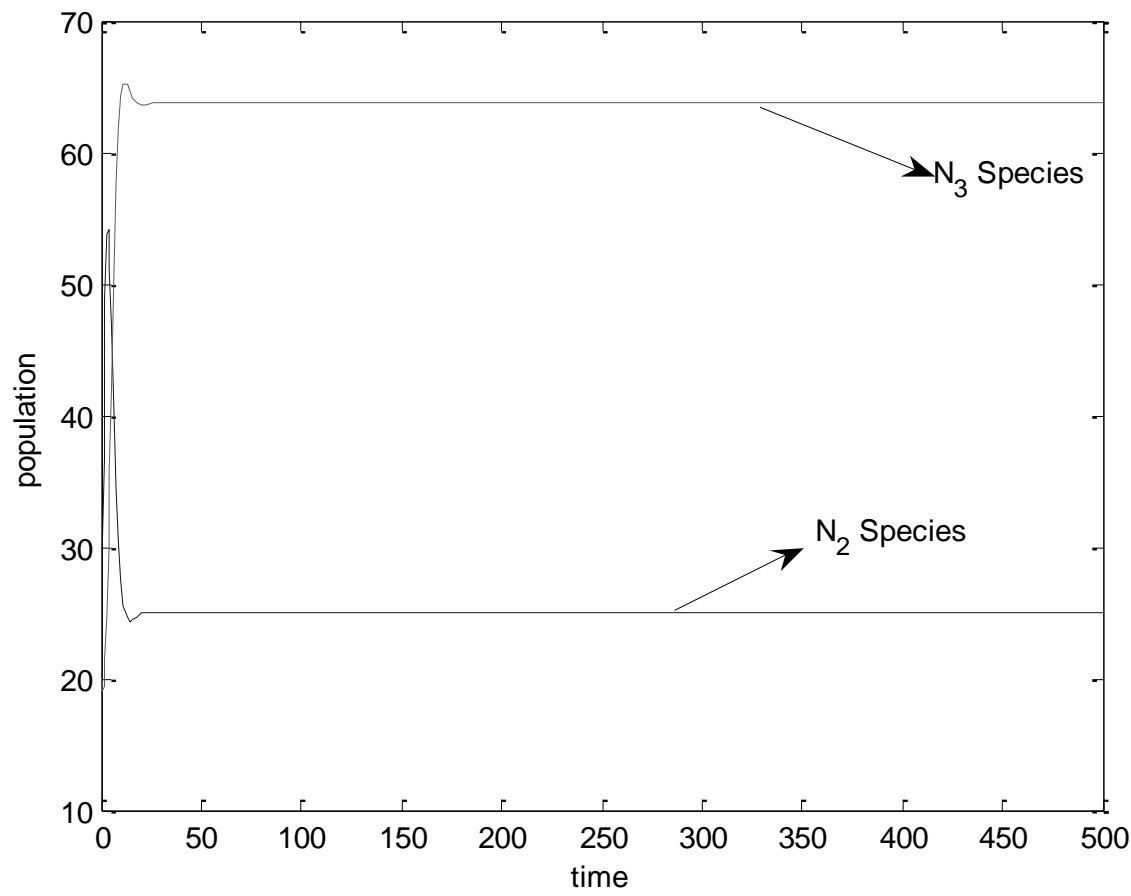

Figure 3. Solution Curves When the Prey Species $\mathbf{N}_{1}$ is Absent

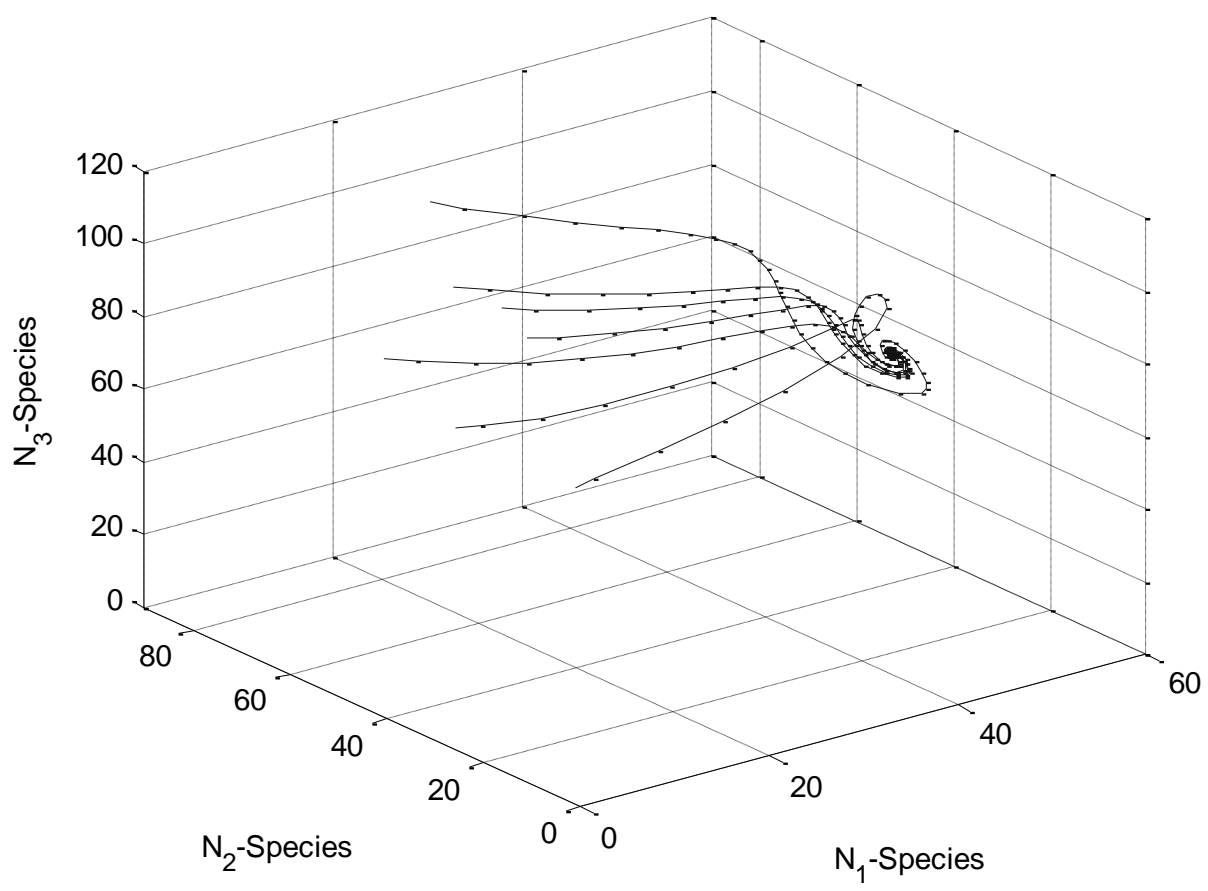

Figure 4. Phase Diagram in Terms of the Values of the Parameters Taken in Example 1

In Figure 1, we see the solution curves which exhibits the stability of the system at $P_{6}$ and in Figure 2, Figure 3 we see that in absence of one prey the other survives in presence of the predator also. Thus, this numerical example supports our hypothesis that the facultative mutualism is not disturbed in the 
presence of the predator. In Figure 4, we see that for the considered values of the parameters the system is also globally stable.

Example 2. We consider the following set of values of parameters for numerical analysis of optimal equilibrium.

$r_{1}=2.09, K_{1}=100, \alpha_{12}=0.1, a_{1}=0.01, q_{1}=0.04, r_{2}=2.5, K_{2}=100, \alpha_{21}=0.2, a_{2}=0.02$, $q_{2}=0.05, \beta_{1}=0.3, \beta_{2}=0.4, d=0.2, p_{1}=10, p_{2}=15, c_{1}=5, c_{2}=6, \delta=0.04$

Then optimal harvesting efforts are, $E_{1 \delta}=13.9632, E_{2 \delta}=32.4487$ and corresponding optimal equilibrium point is $\left(N_{1 \delta}, N_{2 \delta}, N_{3 \delta}\right)=(50.529,6.082,48.823)$. From the Figure 7 and Figure 8, it can be realized that the mutualism between the prey species remains facultative even when optimal harvesting efforts are used.

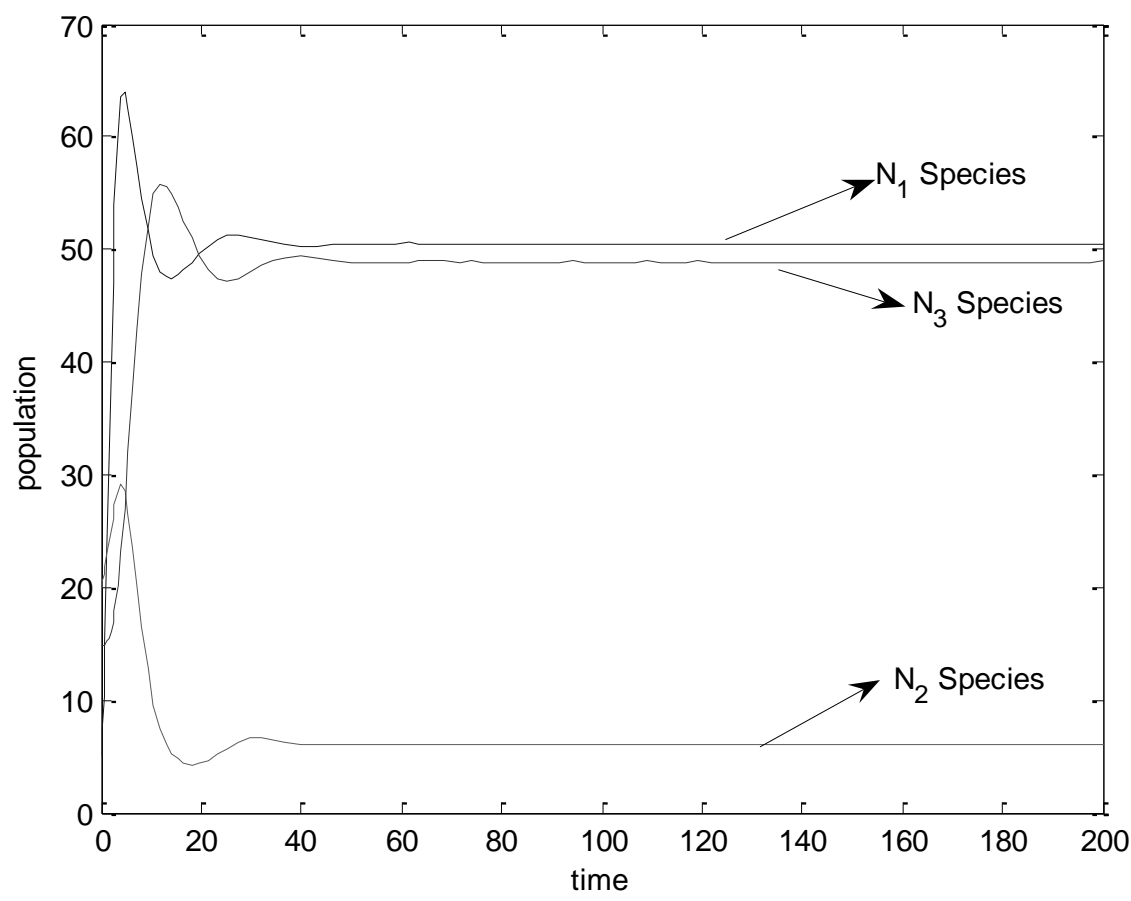

Figure 5. Optimal Solution Curves 


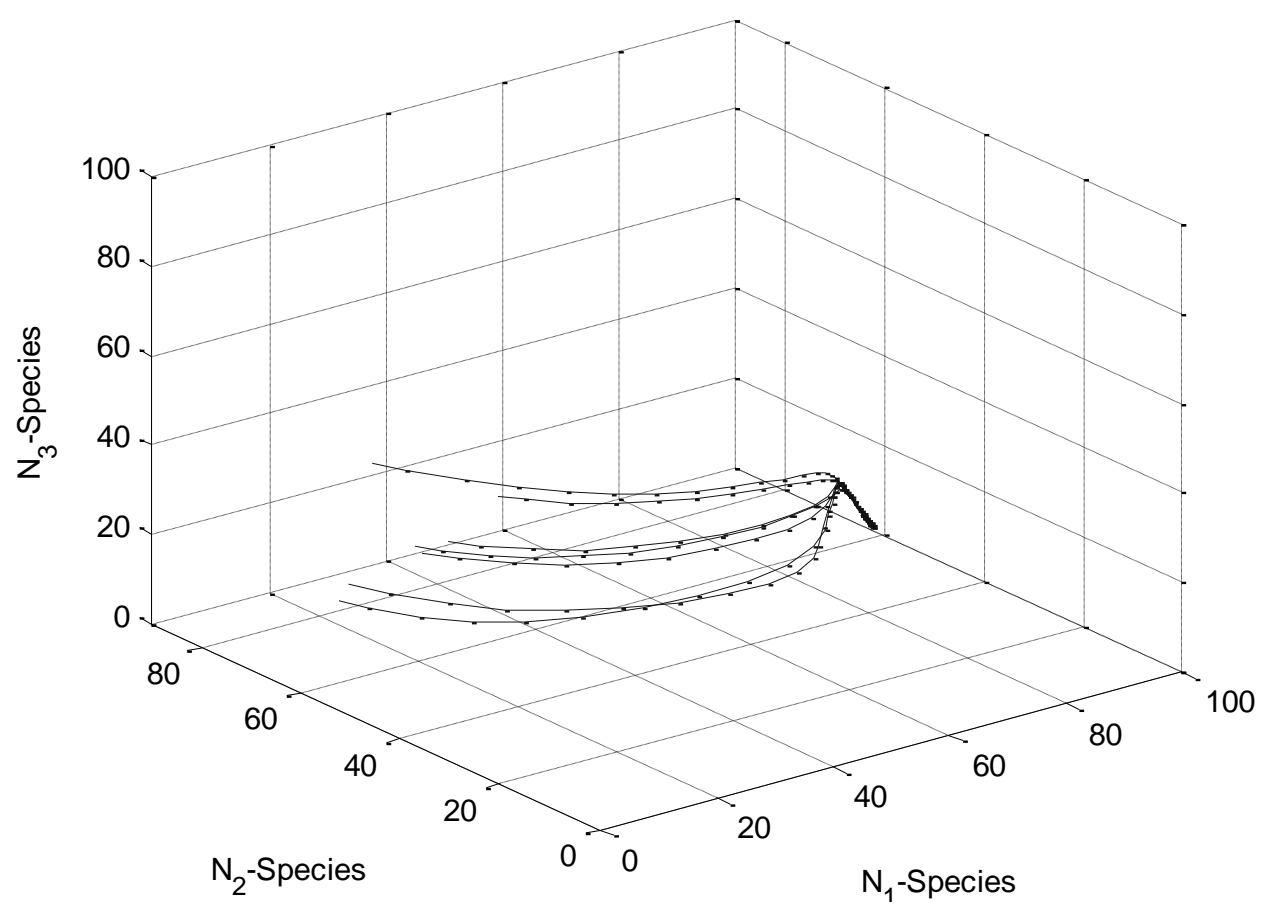

Figure 6. Phase Diagram Corresponding to the Optimal Harvesting Efforts

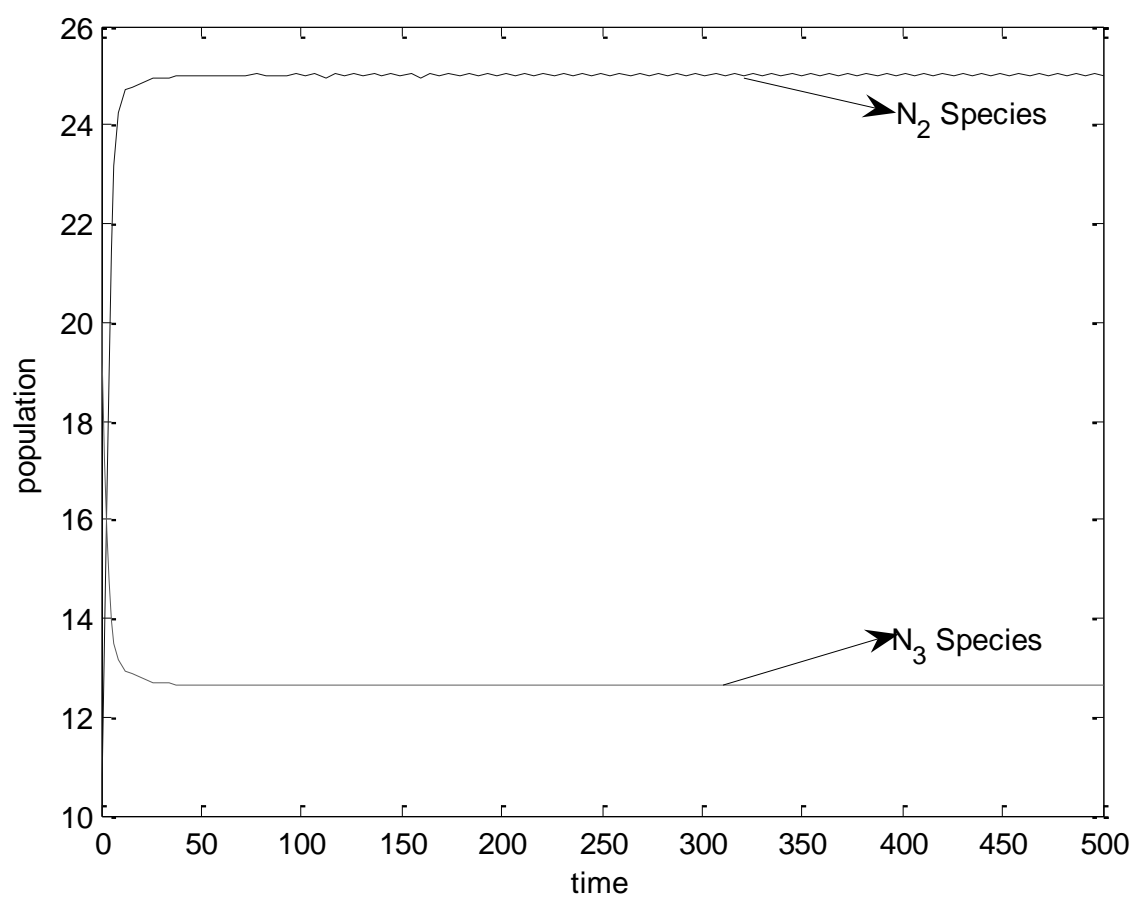

Figure 7. Solution Curves Showing Survival of $\mathbf{N}_{2}$ Species in Absence of $N_{1}$ Species 


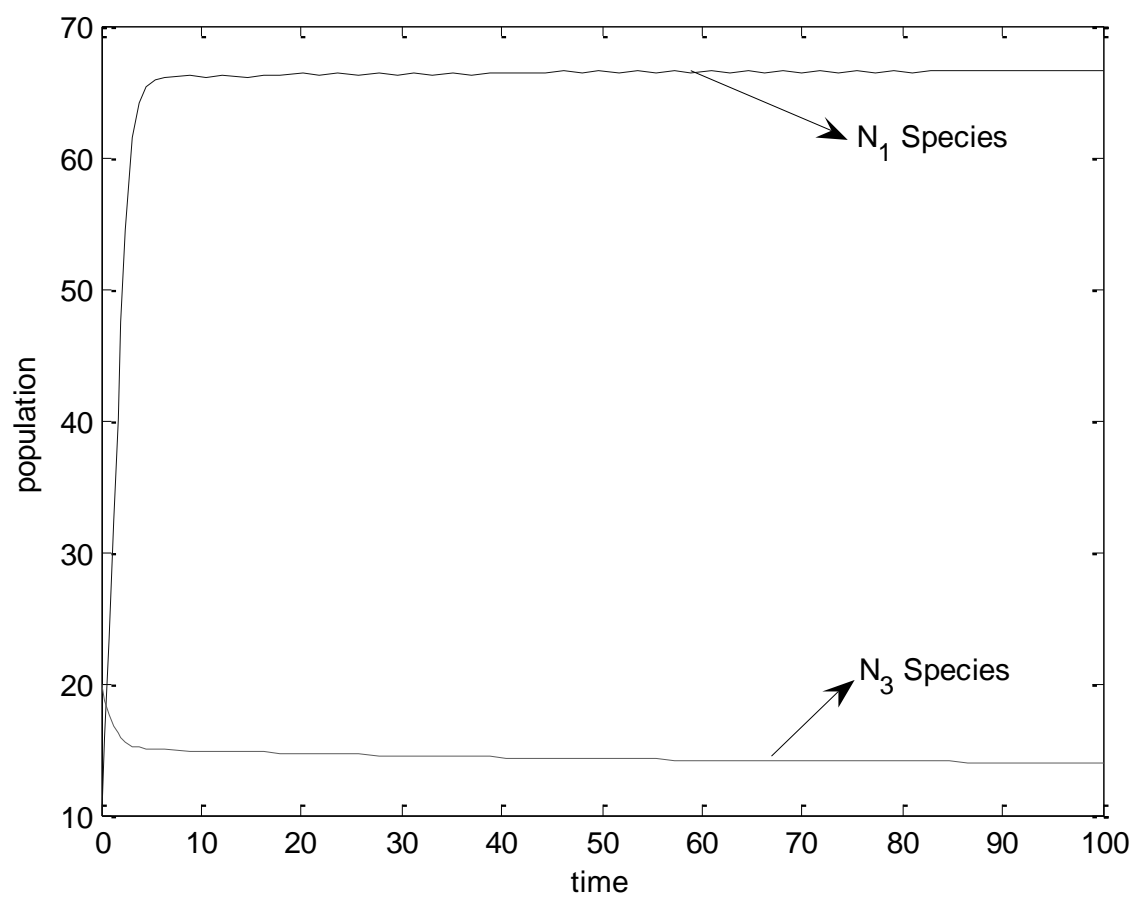

Figure 8. Solution Curves Showing Survival of $\mathbf{N}_{1}$ Species in Absence of $\mathbf{N}_{2}$ Species

\section{Conclusion}

In this paper, we have presented a mutualism model with independent harvesting efforts on mutualist prey species in presence of a predator. During equilibrium analysis and numerical simulation we have seen that the predator survives even when one species is extinct. The important fact is that the mutualism remains facultative in presence of the predator. This is due to the survival of one species in absence of other and in presence of the predator. We derive the optimal harvesting policy and numerically the optimal equilibrium, optimal harvesting efforts are obtained. Numerical illustrations show that, the mutualism remains facultative even when optimal harvesting efforts are used on prey species. This paper includes simple linear predation functions and does not include obligate mutualism between the prey species. We used Mat Lab for numerical calculations and graphs.

\section{Acknowledgement}

The research of Dr. Saroj Kumar Chattopadhyay is financed by the University Grants Commission of India (F.NO.PSW-184/14-15 (ERO)).

\section{References}

Clark, C. W. (1985). Bioeconomic Modelling and Fisheries Management. Wiley, New York.

Clark, C. W. (1990). Mathematical Bioeconomics: The Optimal Management of Renewable Resources. John Wiley \& sons, New York.

Dai, G., \& Tang, M. (1998). Coexistence region and global dynamics of harvested predator-prey system. 
SIAM J. Appl. Math., 13, 193-210. https://doi.org/10.1137/S0036139994275799

Janzen, D. H. (1985). The natural history of mutualisms. In D. H. Boucher (Ed.), The Biology of Mutualism (pp. 40-99). Oxford University Press, Oxford.

Kar, T. K., \& Chaudhuri, K. S. (2004). Harvesting in a two prey one predator system: A bioeconomic model. ANZIAM J., 45, 443-456. https://doi.org/10.1017/S144618110001347X

Kot, M. (2001). Elements of Mathematical Ecology. Cambridge University Press. https://doi.org/10.1017/cbo9780511608520

Lengeler, J. W., Drews, G., \& Schlegel, H. G. (1999). Biology of the Prokaryotes. Blackwel Science, Stuttgart.

Margulis, L. (1970). Origin of Eukaryotic Cells. Yale University Press, New Haven.

Margulis, L. (1981). Symbiosis in Cell Evolution. W. H. Freeman, San Francisco.

May, R. M. (1981). Theoretical Ecology: Principles and Applications. Sinauer Associates, Sunderland, MA.

Mesterton, G. M. (1988). On the optimal policy for combined harvesting of predator and prey. Natural Resources Modelling, 3, 63-90.

Strobele, W. J., \& Wacker, H. (1995). The economics of harvesting predator-prey system. J. Econ., 61, 65-81. https://doi.org/10.1007/BF01231484

Wallin, I. E. (1923).The mitochondria problem. American Naturalist, 57, 255-261. https://doi.org/10.1086/279919

Wallin, I. E. (1927). Symbionticism and the origin of Species. Williams \& Wilkins, Baltimore, MD. https://doi.org/10.5962/bhl.title.11429 